\title{
ON THE DESIGN OF MORTGAGES AND THE NEED FOR INDEXATION
}

\author{
F. C. GAIR'
}

(Received 6 January 1983; revised 12 May 1983)

\begin{abstract}
There is a well-recognized need for a mortgage instrument that will operate satisfactorily in the presence of a volatile inflation. This paper analyses a large class of mortgages - the 'continuous mortgage' (CM) - as a basis for such design. In particular, it is shown that the real (inflation-adjusted) payment stream is exponentially sensitive to changes in the real interest rate. Consequently to realize a satisfactory design, the mortgage must be indexed by assigning the real interest rate. Then, under appropriate restraints, the CM offers a continuum of satisfactory mortgage designs.
\end{abstract}

\section{Introduction}

This paper arose from an attempt to design a mortgage which can be guaranteed to operate satisfactorily - even optimally - in the presence of inflation. From one point of view, the task is impossible, since inflation is unpredictable and may become large and volatile. But from another point of view the task is straight-forward. We need only allow for inflation (or adjust to it after it has occurred) by the process of indexation.

Indexation consists of denominating financial contracts in 'real terms', i.e. in terms of units of constant purchasing power, as measured with reference to 'a suitable commodity basket.' Any quantity expressed relative to this is called 'real', or is expressed 'in real terms'. In contrast, quantities expressed in money terms can be called 'nominal'. Thus indexation cancels out (by definition) after the fact

\footnotetext{
'Department of Mathematics, University of Canterbury, Christchurch 1, New Zealand.

(C) Copyright Australian Mathematical Society 1984, Serial-fee code 0334-2700/84
} 
the major average inflation fluctuations, and leaves only the (usually smaller) 'real' relative price fluctuations.

Throughout this paper we have the consumer price index (CPI) in mind. However, the analysis and conclusions remain valid for any chosen commodity basket, provided inflation is measured relative to that chosen basket.

In fact, many forms of indexation are possible, though acceptance of an index other than the CPI should be made with great caution. For example, the linking of Israeli mortgages to the U.S. dollar had disastrous consequences in 1962 [8, page 160]. On the other hand, while wage indexation clearly has some advantages, these are two-edged-real mortgage payments will reduce if real wages reduce, but they will increase if real wages increase. (Such a wage indexation of mortgage repayments has been an option for low income earners in Brazil [1, page 122].)

As might be expected, many economists believe that the widespread unfavourable effects of inflation can be greatly reduced by indexation [7]. This belief has been reinforced by the successful implementation of indexation in Brazil since 1964 [1] in spite of a high inflation rate ( 100\% per year). However, there has been much resistance against indexation-especially in countries with moderate inflation rates ( $\sim 10 \%$ per year) for which indexation is thought by some to be unnecessary or undesirable.

Rather than attempting any general consideration of arguments for or against indexation [7], [12], we focus our attention on the relevance of indexation to mortgage design. We make the generally accepted assumption that the shape of the stream of repayments in real terms is of primary importance in the design [3], [4], [6], [7], [11], [12].

We begin with a brief summary of the inflation-induced problems associated with the conventional non-indexed mortgage instruments, as described qualitatively by Lessard and Modigliani [6].

The standard mortgage (SM), with its constant (nominal) interest rate and constant (nominal) repayment rate, has proved to be an unsatisfactory instrument during recent years of high and unpredictable inflation for the following reasons. The constant (nominal) interest rate is inappropriate since this rate should be allowed to vary to accommodate anticipated inflation. Moreover, as (nominal) interest rates on new mortgages increase with anticipated inflation, higher initial repayment rates are induced. These may be so high as to become impracticable for the potential home owner. Because the (nominal) repayment rate is constant, the real repayment rate will decline at the inflation rate, producing a 'negative tilt'.

The extent of this tilt is illustrated in Table 1 for mortgages of (typical) 25 year duration, for a range of constant annual inflation rates $\bar{\lambda}_{a}$. (Note that $\left(1+\bar{\lambda}_{a}\right)^{25}$ $=$ ratio of final to initial price levels $=$ ratio of initial to final real payment rates.) 
TABLE 1. Dependence of $\left(1+\bar{\lambda}_{a}\right)^{25}$ on the annual inflation rate $\bar{\lambda}_{a}$

\begin{tabular}{|l|ccccccc|}
\hline $\bar{\lambda}_{a}(\%)$ & 1 & 2 & 5 & 10 & 15 & 20 & 30 \\
\hline$\left(1+\bar{\lambda}_{a}\right)^{25}$ & 1.282 & 1.641 & 3.386 & 10.83 & 32.92 & 95.40 & 705.6 \\
\hline$\lambda(\% /$ year $)$ & .995 & 1.980 & 4.879 & 9.531 & 13.98 & 18.23 & 26.24 \\
\hline
\end{tabular}

(The continuous inflation rate $\lambda=\log _{e}\left(1+\bar{\lambda}_{c}\right)$ is given for comparison-see Section 5.)

We see, for example, that for $\bar{\lambda}_{a}=10 \%$, this ratio is already prohibitively high at 10.83 , and greater values of $\bar{\lambda}_{a}$ render the ratio successively more impossible. Only such small values as $\bar{\lambda}_{a} \sim 1 \%$ could be regarded as corresponding to acceptable payment ratios $(\sim 1.3)$ for the SM. Moreover, in practice $\bar{\lambda}_{a}$ will not remain constant during the mortgage, but will fluctuate unpredictably. Thus the real repayment burden is concentrated in the early years of the SM to produce a highly unsatisfactory instrument which in real terms remains completely vulnerable to the whims of inflation.

The situation has been only marginally alleviated by the use of the standard variable rate mortgage (SVRM) which allows some of the required adjustments in the interest rate to occur. However, each interest rate change is made at the expense of an undesirable discontinuity in the repayment rate. (At each change it is as though a new mortgage is started.) Moreover, the negative tilt in the real repayment rate and its associated problems remain unabated.

These high initial repayment rates dampen the demand for mortgages. Consequently, the real interest rate decreases, which in turn dampens the supply of mortgage finance. In fact such gravely distorted mortgage markets continue to function only under the restraints of ad hoc governmental controls and subsidies.

If housing subsidies are part of government policy then they should be applied in a predictable manner rather than unpredictably as a result of inflation.

A number of innovative alternative mortgage instruments have been proposed as partial or complete solutions of these problems, [2] to [8], [11]. Some of these designs offer marked improvements over the SM and the SVRM. However, it is pertinent that of all these designs only the price level adjusted mortgage (PLAM [4]) is indexed, and only the PLAM has been in successful operation over an extended period [1].

We will argue more generally that only indexed designs should be considered, and we will present a continuum of such designs.

Our conclusions will be based on the quantative study of the class of all mortgages with 'smoothed real payment design' which we call the continuous mortgage $(\mathrm{CM})$. This class contains virtually all of the proposed new mortgage instruments. 
This paper has the following aims:

(1) to present the CM as a continuum of practical mortgage designs

(2) to derive the important quantitative relationships for the CM

(3) to deduce appropriate constraints on the CM to guarantee it as a satisfactory design regardless of the severity or volatility of inflation.

A continuous model is used in our analysis, rather than a discrete one, for the following reasons.

(1) The mathematical analysis of the continuous model is more attractive. It avoids the choice of an arbitrary time period for the compounding of interest needed for the discrete analysis. The relationships between the various rates (interest, inflation, repayment increment, control function) are particularly simple in the continuous model.

(2) Certain deceptive practices are impossible in the continuous model. On the other hand, most banks use a number of different discrete models, with differing time periods, for different purposes. The resulting effective interest rates can be computed [9], but this is likely to be beyond the scope of the layman. Such an environment is wide open to deceptive practices.

(3) Banks have already made some moves towards the continuous model. It is increasingly their practice to calculate interest on certain special purpose and savings accounts on a monthly basis (based on an average daily balance over the month). This is equivalent to compounding 12 times a year. It can be seen from Appendix 2 that this $(m=12)$ is very close to compounding continuously $(m=\infty)$. Such moves towards continuous compound interest should be encouraged.

(4) The widespread use of computers by banks and the availability of relatively cheap electronic calculators to the general public, make use of the continuous calculations an immediate practicable possibility.

The paper is structured as follows. In Section 2, it is convenient to begin by formulating the continuous model of the general mortgage (GM) for the loan function $L$ with variable interest rate $l$, repayment rate $Q$, and duration $T$. In Section 3 , the restriction to a positive continuous $Q$ gives the $\mathrm{CM}$. Here, introduction of the control function $\alpha$ enables us to view the $C M$ as a general dual rate mortgage. The fundamental relationship (10a) between $L$ and $Q$ is derived. In Section 4 , taking $\alpha$ constant gives the simple continuous mortgage (SCM). In Section 5, the analysis is reviewed in real terms. In Section 6 , the qualitative dependence of the CM functions on the dual rates $\alpha, \beta$ or $\alpha, l$ is derived, and the results are detailed in Table 2 . Then the fundamental relationship is illustrated quantitatively for the SCM in Table 3. In Section 7, the factors determining the control and predictability of the real $\mathrm{CM}$ functions are considered quantitatively, and the results appear in Table 4. The imposition of constraint (25a) on $\beta$ is then considered, and its effect is illustrated in Table 5. 
In Section 8 the consequences of the additional constraint $\mathcal{Q} \leqslant R_{0} E_{\gamma_{0}}$ are considered. These result in the inter-related restrictions (29) on $L_{0}$ and $\alpha$, which are illustrated numerically in Tables 6(a), (b), (c).

In Section 9 the conclusions are made.

\section{The general mortgage (GM)}

If a loan $L_{0}$ is borrowed at time 0 at a variable interest rate $l(t)$ at time $t$, and $L_{0}$ is to be fully repaid (with interest) at time $T$, the end of the mortgage period, then the loan $L(t)$ owing at $t$ satisfies the following conditions:

$$
\begin{gathered}
L(0)=L_{0}, \quad L(T)=0, \quad L \text { is continuous, } \\
L^{\prime}(t)=l(t) L(t)-Q(t),
\end{gathered}
$$

wherever $l$ and $Q$ are continuous in the interval $[0, T]$, where $Q$ is the repayment rate function. As already mentioned, as well as assuming that interest accrues continuously, this model requires that the repayments are made continuously, at rate $Q(t)$. (This does not necessarily require $Q$ to be a continuous functionthough this restriction is made in Section 3). In practice it is easy to modify this repayment stream into discrete form without violating the continuous model (see Appendix 1).

Throughout the analysis it is very convenient to introduce the compact notation

$$
E_{a}(t)=\exp \left(\int_{0}^{t} a\right)
$$

for any integrable function $a$. Then $E_{a}$ generalizes the exponential function, retaining many of its properties. For example,

$$
\begin{aligned}
& E_{a} \text { is positive and continuous, and } \\
& E_{a}^{\prime}=a E_{a} \text { wherever } a \text { is continuous. }
\end{aligned}
$$

$E_{a}$ is piecewise exponential if $a$ is a step-function, and exponential if $a$ is constant. Moreover, it is the natural function with which to express the compounding of interest, since unit investment at time 0 compounds to $E_{l}(t)$ at time $t$ when $l(t)$ is the variable interest rate at time $t$.

Now the solution of (1) can be written as

$$
L(t)=E_{l}(t)\left(L_{0}-\int_{0}^{t} E_{-l} Q\right)=E_{l}(t) \int_{t}^{T} E_{-l} Q
$$

where $Q$ is arbitrary except for the constraint

$$
L_{0}=\int_{0}^{T} E_{-l} Q
$$


We will refer to this as the general mortgage (GM). It is clear from (5) that $Q$ can be determined in advance only if $l$ is so specified.

\section{The continuous mortgage (CM)}

Let us now restrict $Q$ to be positive, continuous, and piecewise continuously differentiable. Then we can write

$$
Q(t)=R E_{r}(t)
$$

where $R$, a positive constant, is the initial repayment rate $Q(0)$, and $r$, a piecewise continuous function, the "repayment increment rate", is given by

$$
r(t)=Q^{\prime}(t) / Q(t)
$$

wherever $Q$ is continuously differentiable. Thus $Q$ determines the pair $(R, r)$, and vice versa. In particular $R, r$ determine the initial value, and the shape of $Q$, respectively.

Substitution of (6) into (5) gives

$$
L_{0}=R \int_{0}^{T} E_{r-l}
$$

so that $R$ is determined (and the mortgage can proceed in practice) only when the difference function $l-r$ is specified at the outset for the whole duration of the mortgage. It is therefore important to isolate and name this function.

We therefore define

$$
\alpha=l-r, \text { so that } r=l-\alpha,
$$

and call $\alpha$ the "control function". Thus we normally regard $\alpha$ as being prescribed in advance. (It is possible to allow changes in the prescription of $\alpha$ during the mortgage. However these cause discontinuities in 2 , and produce a piecewise continuous mortgage (which is not considered here).)

If we also define

$$
G_{\alpha}(t)=E_{\alpha}(t) \int_{t}^{T} E_{-\alpha}=\int_{t}^{T}\left[\exp \left(\int_{t}^{u}(-\alpha)\right)\right] d u, \quad 0 \leqslant t \leqslant T
$$

then for each arbitrary (piecewise continuous function) $\alpha$, using (4b), (6), (7), (8) and (9), we deduce the fundamental relationship

$$
L(t)=Q(t) G_{\alpha}(t)
$$

where

$$
Q(t)=R E_{l-\alpha}(t)
$$


and

$$
R=L_{0} / G_{\alpha}(0) .
$$

We will refer to (10) as the "continuous mortgage" (CM). The simple connection (10a) between $L$ and $Q$ shows that $Q / L=1 / G_{\alpha}$ is independent of $l$ and depends only on $\alpha$. It also indicates that $Q$ and $L$ are related at time $t$ exactly as for a new mortgage of duration $T-t$ starting at time $t$. From (9) we find further that

$$
\begin{gathered}
G_{\alpha}(T)=0, \quad G \text { is continuous, } \\
G_{\alpha}(t)>0 \quad \text { for } 0 \leqslant t<T \\
G_{\alpha}^{\prime}(t)=\alpha(t) G_{\alpha}(t)-1 \quad \text { wherever } \alpha \text { is continuous, }
\end{gathered}
$$

so that $G_{\alpha}$ is also a mortgage loan function (like $L$ ) but with interest rate $\alpha$, repayment rate $=1$, and physical dimension of time. Thus $G_{\alpha}$ is a "normalized loan function". It plays a key role in CM design.

\section{The simple continuous mortgage (SCM)}

The simplest case of the $\mathrm{CM}$ is achieved by choosing

$$
\alpha=\text { constant. }
$$

We refer to this case as the simple continuous mortgage (SCM). We now call $\alpha$ the "control parameter". Here (9) reduces to

$$
G_{\alpha}(t)= \begin{cases}\frac{1}{\alpha}\left[1-e^{-\alpha(T-t)}\right] & \text { if } \alpha \neq 0, \\ T-t & \text { if } \alpha=0\end{cases}
$$

which has exactly the form of $L$ for the standard mortgage with interest rate $\alpha$, and unit repayment rate, whereas $(10 \mathrm{c})$ reduces to

$$
R= \begin{cases}\frac{\alpha L_{0}}{1-e^{-\alpha T}} & \text { if } \alpha \neq 0, \\ \frac{L_{0}}{T} & \text { if } \alpha=0\end{cases}
$$

The SM is thus a special case of the SCM with $\alpha=l=$ constant. 


\section{Analysis in real terms}

In order to evaluate any mortgage instrument during times of substantial or variable inflation, it is essential to recast the analysis in real terms, that is to make allowance for the changes in the real value of money due to inflation.

If $\lambda$ is the (continuous model) inflation rate function, then $E_{\lambda}$ is the price level function (i.e. $E_{\lambda}(t)$ is the price at $t$ of goods having unit price at time 0 ). Let

$$
\mathcal{L}=E_{-\lambda} L, \quad 2=E_{-\lambda} Q, \quad \beta=l-\lambda .
$$

Then $\mathcal{L}, \mathcal{Q}, \beta$ are respectively the loan, repayment rate, and interest rate functions in real terms (i.e. inflation adjusted to the price level at $t=0$ ). We will see that all conditions between the 'nominal mortgage functions' $L, Q, l, r$ are easily translated into conditions between the corresponding 'real mortgage functions' $\mathcal{L}, 2, \beta, \gamma$ with the 'neutral mortgage functions' $\alpha, E_{\alpha}, G_{\alpha}$ remaining unchanged throughout.

Thus in real terms, for the general mortgage we get

$$
\begin{gathered}
\mathscr{E}(0)=L_{0}, \quad \mathcal{L}(T)=0, \quad \mathcal{L} \text { is continuous, } \\
\mathcal{L}^{\prime}(t)=\beta(t) \mathscr{L}(t)-\mathscr{L}(t),
\end{gathered}
$$

wherever $\beta$ and 2 are continuous in $[0, T]$,

$$
\mathcal{L}(t)=E_{\beta}(t)\left(L_{0}-\int_{0}^{t} E_{-\beta} \mathcal{Q}\right)=E_{\beta}(t) \int_{t}^{T} E_{-\beta} \mathcal{Q},
$$

where 2 is arbitrary except for the constraint

$$
L_{0}=\int_{0}^{T} E_{-\beta} \mathcal{Q},
$$

so that 2 can be determined in advance only if $\beta$ is so specified. For the continuous mortgage, the 'fundamental relationship' (10a) becomes

$$
\mathcal{E}(t)=\mathcal{2}(t) G_{\alpha}(t),
$$

where

$$
\mathcal{2}(t)=R E_{\beta-\alpha}(t)=R E_{\gamma}(t),
$$

and

$$
\gamma=\beta-\alpha=r-\lambda=2^{\prime} / 2
$$

is the repayment increment rate in real terms, and $R$ is given by $(10 \mathrm{c})$. Note that the fundamental relationship (10a) or (19a) can be rewritten as

$$
Q(t) / L(t)=2(t) / \mathscr{L}(t)=1 / G_{\alpha}(t),
$$

which depends only on $\alpha$ and $t$ (and is independent of $\beta, l$, or $\lambda$ ).

As before the SCM is the special case when $\alpha$ is constant. 
The relationship between the GM, CM, SCM, SM, and PLAM [3], [4] are conveniently illustrated in Figure 1, the downward progression indicating successive specializations.

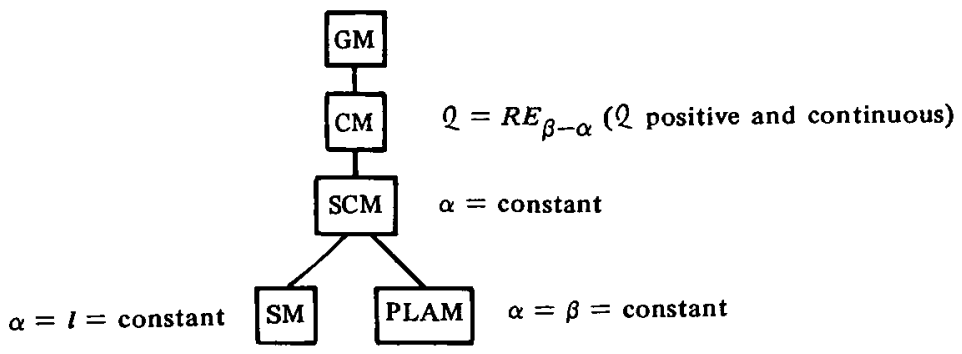

FIGURE 1

The variable rate graduated payment mortgage (VRGPM) of Tucker [11] appears to be the discrete model analogue of the SCM, and the constant payment factor variable rate mortgage (CPFVRM) [3], [4] is a somewhat more complicated approximation of this.

It is clear from $(19 \mathrm{c}, \mathrm{f})$ that $\gamma$ determines the tilt of 2 which is $\gtrless 0$ according as $\gamma \gtrless 0$. Since $2(t) / R=E_{\gamma}(t)=E_{\beta-\alpha}(t)$ we have the straightforward result that $\mathcal{Q}(t) / R$ is precisely determined by $\beta-\alpha$. This simple result is in striking contrast with the conclusion of Cohn and Fischer [4, page 67] that the CPFVRM had 'a real payment that showed no clear trend over the 1951-70 period' which was chosen to illustrate the design. It is of interest to note that for the PLAM, $\mathcal{2}(t) / R=1$, so that 2 then has zero tilt, whereas for the SM, $2(t) / R=E_{-\lambda}(t)$ so that the negative tilt depends directly on $\lambda$. (This has already been illustrated in Table 1 (Section 1) for $t=T=25$ years, and $\lambda=$ constant.)

The question of designing a CM with 2 of arbitrary tilt, and more generally of arbitrary shape, is considered in Sections 6, 7,8.

\section{CM dependence on dual rates}

The qualitative dependence of the functions of the $\mathrm{CM}$ on the dual rates are illustrated in Table 2.

We say, for example, that $E_{\alpha}$ increases relative to $\alpha$, and write $E_{\alpha}$ inc $(\alpha)$, since

$$
E_{\alpha_{1}}(t)=\exp \int_{0}^{t} \alpha_{1} \leqslant \exp \int_{0}^{t} \alpha_{2}=E_{\alpha_{2}}(t) \text { for } 0 \leqslant t \leqslant T
$$


provided $\alpha_{1}(t) \leqslant \alpha_{2}(t)$ for $0 \leqslant t \leqslant T$, which we abbreviate to $E_{\alpha_{1}} \leqslant E_{\alpha_{2}}$ provided $\alpha_{1} \leqslant \alpha_{2}$. Similarly the remaining increasing (inc) or decreasing (dec) variations indicated in the Table follow from the monotonicity of the integral and of exp.

TABLE 2. CM dependence on the dual rates (for fixed $L_{0}, T$ ).

\begin{tabular}{|c|c|c|c|c|}
\hline Type & Function defined on $[0, T]$ & $\alpha$ & $\beta$ & $l$ \\
\hline Neutral & $\begin{array}{l}E_{\alpha}(t)=\exp \int_{0}^{t} \alpha \\
G_{\alpha}(t)=\int_{t}^{T}\left[\exp \left(\int_{t}^{u}(-\alpha)\right)\right] d u \\
\frac{Q(t)}{L(t)}=\frac{2(t)}{\mathcal{E}(t)}=\frac{1}{G_{\alpha}(t)} \\
R=\frac{L_{0}}{G_{\alpha}(0)}\end{array}$ & $\begin{array}{l}\text { inc } \\
\text { dec }\end{array}$ & $\begin{array}{l}- \\
-\end{array}$ & $\begin{array}{l}- \\
-\end{array}$ \\
\hline Real & $\begin{array}{l}\gamma=\beta-\alpha \\
\mathcal{Q} / R=E_{\gamma}=E_{\beta-\alpha} \\
\mathcal{Q}=R E_{\gamma}=\frac{L_{0}}{G_{\alpha}(0)} E_{\beta-\alpha} \\
\mathcal{E}=R E_{\beta-\alpha} G_{\alpha}=L_{0} E_{\beta-1 / G_{\alpha}}\end{array}$ & $\begin{array}{l}\text { dec } \\
\text { dec } \\
\text { dec } \\
\text { tilt } \\
\text { dec }\end{array}$ & $\begin{array}{l}\text { inc } \\
\text { inc } \\
\text { inc } \\
\text { inc }\end{array}$ & $\begin{array}{l}- \\
- \\
-\end{array}$ \\
\hline Nominal & $\begin{array}{l}r=l-\alpha \\
Q / R=E_{r}=E_{l-\alpha} \\
Q=R E_{r}=\frac{L_{0}}{G_{a}(0)} E_{l-\alpha} \\
L=R E_{l-\alpha} G_{\alpha}=L_{0} E_{l-1 / G_{\alpha}}\end{array}$ & $\begin{array}{l}\text { dec } \\
\text { dec } \\
\text { dec } \\
\text { tilt } \\
\text { dec }\end{array}$ & $\begin{array}{l}- \\
- \\
-\end{array}$ & $\begin{array}{l}\text { inc } \\
\text { inc } \\
\text { inc } \\
\text { inc }\end{array}$ \\
\hline
\end{tabular}

We see that $2, \mathcal{L}$ both increase with $\beta$ through the factor $E_{\beta}$ which is of 'exponential type' and is therefore very sensitive to $\beta$. This signals the need for controlling fluctuations in $\beta$ (as discussed in Section 7).

The dependence of $2, \mathcal{E}$ on $\alpha$ is more subtle. As a function, $\mathcal{Z}$ is neither increasing nor decreasing relative to $\alpha$. However increasing $\alpha$ decreases the tilt of 2 (or increases its negative tilt). This can be argued as follows:

Now $2=R E_{\gamma}=L_{0} E_{\beta-\alpha} / \int_{0}^{T} E_{-\alpha}$ and let $2+\delta \mathscr{2}=L_{0} E_{\beta-\alpha-\delta \alpha} / \int_{0}^{T} E_{-\alpha-\delta \alpha}$ be the function corresponding to 2 when $\alpha$ is changed to $\alpha+\delta \alpha$, where $\delta \alpha$ is an arbitrary positive incremental function. Then $E_{-\delta \alpha}(u)$ is continuous and decreasing relative to $u \in[0, T]$. It follows that

$$
\begin{gathered}
E_{-\delta \alpha}(T) \leqslant E_{-\delta \alpha}(u) \leqslant 1, \\
E_{-\delta \alpha}(T) E_{-\alpha}(u) \leqslant E_{-\alpha-\delta \alpha}(u) \leqslant E_{-\alpha}(u),
\end{gathered}
$$


and

$$
E_{-\delta \alpha}(T) \int_{0}^{T} E_{-\alpha} \leqslant \int_{0}^{T} E_{-\alpha-\delta \alpha} \leqslant \int_{0}^{T} E_{-\alpha} .
$$

Hence

$$
\int_{0}^{T} E_{-\alpha-\delta \alpha}=E_{-\delta \alpha}(v) \int_{0}^{T} E_{-\alpha} \text { for some } v \in(0, T),
$$

so that

$$
(2+\delta \mathscr{Q})(t)=\mathcal{Q}(t) E_{-\delta \alpha}(t) / E_{-\delta \alpha}(v) \gtrless \mathcal{Q}(t) \text { according as } t \lessgtr v .
$$

Hence

$$
\mathscr{2}(t) \operatorname{inc}(\alpha) \text { for } 0 \leqslant t<v
$$

and

$$
\mathcal{2}(t) \operatorname{dec}(\alpha) \text { for } v<t \leqslant T .
$$

We describe this by saying that increasing $\alpha$, decreases the tilt of 2 (or increases its negative tilt). The variable 'pivot point' $(v, 2(v))$ depends on $\alpha$ and $\delta \alpha$.

Since a similar analysis holds when $2, \gamma, \beta$ are replaced by $Q, r, l$, an analogous tilting of $Q$ occurs when $\alpha$ is increased (with $l$ held fixed).

In order to show that $\mathcal{L} \operatorname{dec}(\alpha)$, the following form of $\mathscr{L}$ is required. From $(16 a, d)$ and $(19 a)$, it follows that

$$
\mathcal{L}^{\prime}(t)=\left(\beta(t)-1 / G_{\alpha}(t)\right) \mathcal{L}(t) \text { for } 0 \leqslant t<T,
$$

so that

$$
\mathcal{L}=L_{0} E_{\beta-1 / G_{\alpha}}
$$

from which it follows that $\mathcal{L} \operatorname{dec}(\alpha)$. Similarly from (la,d) and (10a)

$$
\begin{gathered}
L^{\prime}(t)=\left(l(t)-1 / G_{\alpha}(t)\right) L(t) \text { for } 0 \leqslant t<T, \\
L=L_{0} E_{l-1 / G_{\alpha}},
\end{gathered}
$$

and $L \operatorname{dec}(\alpha)$ also. (Note that as $t \rightarrow T-, G_{\alpha}(t) \rightarrow 0+, E_{-1 / G_{\alpha}}(t) \rightarrow 0$, so that $\mathcal{L}(t)$ and $L(t) \rightarrow 0$ as required.)

From (20), (21) we can judge the extent to which $\mathcal{E}$ or $L$ is increasing (or decreasing) relative to $t$ merely by comparing $1 / G_{\alpha}$ with $\beta, l$ respectively. Also the ratio of repayment rate to interest repayment rate is given in real or nominal terms by

$$
2 / \beta \varrho=1 / \beta G_{\alpha}, \quad Q / l L=1 / l G_{\alpha},
$$

respectively. If inflation is substantial, there should be little concern if $L$ is increasing during the early mortgage years provided $\mathcal{L}$ is decreasing at a sufficient rate. 
For the SCM, since $\alpha$ is constant, the fundamental relationship can be illustrated quantitatively. This is done in Table 3, which gives the dependence of $Q(t) / L(t)=2(t) / \mathscr{L}(t)=1 / G_{\alpha}(t)$ on $\alpha$ and $T-t$. For $t=0$, Table 3 gives values of $R / L_{0}=1 / G_{a}(0)$.

TABLE 3. Repayment rate per year as fraction of outstanding loan (for SCM).

Values per year of $Q(t) / L(t)=2(t) / L(t)=1 / G_{\alpha}(t)$

\begin{tabular}{|c|c|c|c|c|c|c|c|c|}
\hline $\begin{array}{l}\alpha \quad T-t \text { in } \\
\text { in years } \\
\% / \text { year }\end{array}$ & 5 & 10 & 15 & 20 & 25 & 30 & 40 & $\infty$ \\
\hline 0 & .2000 & .1000 & .0667 & .0500 & .0400 & .0333 & .0250 & .0000 \\
\hline 2 & .2102 & .1103 & .0772 & .0607 & .0508 & .0443 & .0363 & .0200 \\
\hline 4 & .2207 & .1213 & .0887 & .0726 & .0633 & .0572 & .0501 & .0400 \\
\hline 6 & .2315 & .1330 & .1011 & .0859 & .0772 & .0719 & .0660 & .0600 \\
\hline 8 & .2427 & .1453 & .1145 & .1002 & .0925 & .0880 & .0834 & .0800 \\
\hline 10 & .2542 & .1582 & .1287 & .1157 & .1089 & .1052 & .1019 & .1000 \\
\hline 12 & .2660 & .1717 & .1438 & .1320 & .1263 & .1234 & .1210 & .1200 \\
\hline 14 & .2781 & .1858 & .1529 & .1491 & .1444 & .1421 & .1405 & .1400 \\
\hline 16 & .2906 & .2005 & .1760 & .1668 & .1630 & .1613 & .1603 & .1600 \\
\hline 18 & .3033 & .2156 & .1930 & .1851 & .1820 & .1808 & .1801 & .1800 \\
\hline
\end{tabular}

Taking $t=0$ we see that for a long term mortgage ( $T \geqslant 20$ years say), practicable values of $R / L_{0}(\leqslant 10.02 \%$ per year, say) can only be achieved by choosing sufficiently small $\alpha$ ( $\leqslant 8 \%$ per year, say). This desirable possibility is removed if we are restricted to the SM (with $\alpha=l$ ) whenever $l$ is moderately large ( $>8 \%$ per year, say). For example if $l=18 \%$ per year, $T=25$ years, we can compare an SCM with $\alpha=8 \%$ per year giving $R / L_{0}=9.25 \%$ per year with an SM (with $\alpha=l=18 \%$ per year) giving $R / L_{0}=18.20 \%$ per year which is impracticably high. Bounds for $\alpha$ are considered more generally in Section 8 .

For the SCM, Table 3 also enables quick calculation of $Q^{\prime} / e, L^{\prime} / L, 2 /(\beta \complement)$, $Q /(l L)$ for any value of $t, \alpha$, and $T$ (using (20), (21), (22)). Table 3 is also useful in SCM design, as illustrated in Tables 6(a), (b), (c) (Section 8).

\section{Control and predictability of the real CM functions}

From Table 2 we see that for fixed $L_{0}, T$ the real CM functions are determined by the dual rates $\alpha, \beta$. The function $\alpha$ is entirely at the designer's disposal, whereas the function $\beta$ normally depends on the money market. 
If the function $\beta$ is predictable (e.g. for $\beta=$ constant $-a$ fixed rate price level adjusted mortgage (PLAM)), then by choosing $\alpha=\beta-\gamma, \gamma$ arbitrary, we can effect a $\mathrm{CM}$ with $2=R E_{\gamma}$ of any desired shape whatsoever, since this shape is determined by $\gamma$ alone.

However, if the function $\beta$ is unpredictable (e.g. for a variable rate mortgage, or for a fixed nominal rate mortgage in the presence of inflation), this unpredictability is inevitably transmitted to the real CM functions. The exact measure of this effect is shown in Table 4.

TABLE 4. Measures of real CM function predictability

\begin{tabular}{|c|c|c|c|c|c|c|}
\hline Type & $\mathrm{CM}$ & $\begin{array}{c}\text { Formula } \\
\text { for } \alpha\end{array}$ & $\begin{array}{c}\text { Prescription } \\
\text { for } \alpha\end{array}$ & $\begin{array}{c}\text { Deviation } \\
\gamma-\gamma_{p}\end{array}$ & $\begin{array}{c}\text { Deviation } \\
\text { factor } \\
E_{\gamma}-\gamma_{p}\end{array}$ & Examples \\
\hline 1 & $\beta$ prescribed & $\beta-\gamma$ & $\beta-\gamma_{p}$ & 0 & 1 & $\begin{array}{c}\text { PLAM } \\
(\gamma=0) \\
\text { SM } \\
\left(\lambda_{e}=0\right) \\
\text { SCM } \\
(\alpha=\text { const })\end{array}$ \\
\hline
\end{tabular}

We say that a function is 'prescribed' when its values for the duration of the mortgage are known in advance at $t=0$. In order to determine $R$ through (10c) so that the $\mathrm{CM}$ can proceed, $\alpha$ must be prescribed.

Let $\gamma_{p}$ be the 'target' real repayment increment rate function, and corresponding to a given prescribed $\alpha$, let

$$
\mathcal{Q}_{p}=R E_{\gamma_{p}}, \quad \mathcal{L}_{p}=\mathscr{2}_{p} G_{\alpha},
$$

be the 'target' 2 and $\mathcal{L}$ respectively. Note that the target functions $\gamma_{p}, \mathscr{Q}_{p}, \mathscr{L}_{p}$ are independent of $\beta$, and are at the designer's disposal. In particular $\gamma_{p}$ (and hence the shape of $2_{p}$ ) can be chosen arbitrarily as any integrable function here. We will refer to the target functions $\gamma_{p}, \mathscr{2}_{p}, E_{p}$ collectively as the 'target design'.

Only when $\beta$ is predictable can the condition $\gamma=\gamma_{p}$ be achieved, giving 2 the target shape $2_{p}$. The unpredictability of $\gamma, 2$, and $\mathcal{L}$ are indicated by the 'deviation' $\gamma-\gamma_{p}$, and the 'deviation factor'

$$
2 / 2_{p}=\mathcal{L} / \mathcal{L}_{p}=E_{\gamma-\gamma_{p}}
$$

with its very sensitive exponential dependence on $\gamma-\gamma_{p} . \lambda_{e}$ and $\beta_{e}$ are forward estimates (which must be made at $t=0$ ) of the functions $\lambda, \beta$ respectively.

Types 1 and 2 are special cases of type 3 when $\beta, l$ respectively are frozen.

Type 1 trades the disadvantage of inflexible $\beta$ for the advantage of complete predictability of the real mortgage functions. $\gamma_{p}=0$ gives the PLAM. However, if 
$\gamma_{p}$ is negative, then

$$
\mathcal{Q}(t)=R E_{\gamma_{p}}(t),=R \exp \left(\gamma_{p} t\right) \text { if } \gamma_{p} \text { is constant, }
$$

so that the degree of negative tilt can be chosen at will, depending on the magnitude of $\gamma_{p}$. For example, if there is doubt that real wages can be sustained, then a small negative $\gamma_{p}$ (e.g. $-(1$ or 2$) \%$ per year) could be taken to ensure that mortgage payments will be unlikely to increase as a fraction of wages.

Another possibility would be to inndex the mortgage payments to a wage index, though as previously mentioned this has the two-edged consequence that real mortgage repayments would increase or decrease according as real wages increase or decrease.

Type 2 suffers from the disadvantages both of an inflexible $l$ and also of very unpredictable values of the real mortgage functions, since the deviation $\lambda_{e}-\lambda$ is likely to be large owing to the difficulty of predicting $\lambda$ over the duration of a mortgage. In particular for the SM, the unsuitable estimate $\lambda_{e}=0$ is effectively made giving the deviation factor $E_{-\lambda}$ which is likely to produce a large negative tilt (as previously illustrated in Table 1).

Type 3 allows flexibility of $\beta$ at the expense of the deviation factor $E_{\gamma-\gamma_{p}}=$ $E_{\beta-\beta_{e}}$. Since this factor has very sensitive exponential dependence on $\beta-\beta_{e}$ it is necessary that it should be contained by imposing bounds on $\beta-\beta_{e}$.

If we impose the condition

$$
\left|\beta-\beta_{e}\right| \leqslant \varepsilon \quad \text { where } \beta_{e}, \varepsilon \text { are constants, }
$$

so that

$$
\left|\gamma-\gamma_{p}\right| \leqslant \varepsilon
$$

then

$$
E_{-\varepsilon} \leqslant E_{\beta-\beta_{e}} \leqslant E_{\varepsilon} .
$$

In particular

$$
e^{-\varepsilon T} \leqslant E_{\beta-\beta_{e}}(T) \leqslant e^{\varepsilon T},
$$

so that $e^{\varepsilon T}$ is a measure of the largest possible deviation factor. On the other hand, assuming that $\beta_{e}>\varepsilon$, the ratio of largest to smallest possible $\beta$,

$$
\beta_{\max } / \beta_{\min }=\left(\beta_{e}+\varepsilon\right) /\left(\beta_{e}-\varepsilon\right),
$$

can be regarded as a measure of the flexibility in $\beta$, enabling $\beta$ to change in response to fluctuations in the supply-demand for real money. The dependence of $e^{\varepsilon T}$ and $\beta_{\max } / \beta_{\min }$ on $\varepsilon$ and $\beta_{e}$ is illustrated in Table 5 for $T=25$ years. 
TABLE 5. Values of $\beta_{\max } / \beta_{\min }$ and $e^{e T}$ for $T=25$ years for $\mathrm{CM}$.

\begin{tabular}{|c|c|c|c|c|c|}
\hline $\begin{array}{l}\beta_{e} \varepsilon(\% / \mathrm{yr}) \\
(\% / \mathrm{yr})\end{array}$ & 0 & 0.5 & 1 & 1.5 & 2 \\
\hline 2 & 1 & $1.6 \dot{6}$ & 3 & 7 & $\infty$ \\
\hline 3 & 1 & 1.4 & 2 & 3 & 5 \\
\hline 4 & 1 & 1.29 & $1.6 \dot{6}$ & 2.25 & 3 \\
\hline \hline$e^{e^{T}}$ & 1 & 1.133 & 1.284 & 1.455 & 1.649 \\
\hline
\end{tabular}

A choice of $\beta_{e} \sim 3 \%$ per year, $\varepsilon=1$ or $1.5 \%$ per year would be a reasonable compromise between keeping $e^{\varepsilon T}$ sufficiently small, and allowing $\beta_{\max } / \beta_{\min }$ sufficiently large.

As with type 1 , a small negative bias can be given to $\gamma_{p}$ to counteract any possible decrease in real wages. Since $\alpha=\beta_{e}-\gamma_{p}$, we see that the CM target design is determined by $\alpha$, or equivalently by $\gamma_{p}$. We consider appropriate bounds for $\alpha$ and $\gamma_{p}$ in Section 8.

When $\varepsilon=0$, type 3 reduces to type 1 . Because of this, and because type 2 appears unattractive, we confine further attention to type 3 .

In view of the sensitivity of the deviation factor to $\beta-\beta_{e}$ (for type 3 mortgages), any necessary changes in $\beta$ (within the prescribed limits (25a)) should be controlled by an independent (e.g. government) agency.

\section{Design constraints for the $\mathrm{CM}$}

The design of any mortgage must be compatible with the borrower's ability to repay. Hence we now consider the effect of imposing an upper bound function $\mathscr{2}_{0}$ on 2 . More precisely, for a given $T$, under the assumption that $\beta$ is restricted by (25a) (i.e. for a type $3 \mathrm{CM}$ ), we consider design constraints for the $\mathrm{CM}$ to ensure that

$$
\mathcal{2} \leqslant R_{0} E_{\gamma_{0}}=\mathcal{Q}_{0}
$$

or equivalently

$$
R \leqslant R_{0}, \quad \gamma \leqslant \gamma_{0},
$$

for given upper bounds $R_{0}$ (a constant) and $\gamma_{0}$ (a given function).

$$
\text { Let } L_{0_{\max }}=R_{0} G_{\alpha_{\min }}(0) \text { where } \alpha_{\min }=\beta_{e}+\varepsilon-\gamma_{0} \text {. }
$$


Then in view of (10c), (19d), (25a) and the monotonicity of $G_{\alpha}$ (Table 2), (27) will be satisfied provided we choose

$$
L_{0} \leqslant L_{0_{\max }}
$$

and contain $\alpha$ by

$$
\alpha_{\min } \leqslant \alpha \leqslant \alpha_{\max },
$$

where $\alpha_{\max }$ is uniquely determined by

$$
G_{\alpha_{\max }}(0)=\frac{L_{0}}{R_{0}}, \text { and } \alpha_{\max }=\alpha_{\min }+c,
$$

where $c$ is a constant. Then

$$
G_{\alpha \max }(0) \leqslant G_{\alpha}(0) \leqslant G_{\alpha \min }(0),
$$

or

$$
\frac{L_{0}}{R_{0}} \leqslant \frac{L_{0}}{R} \leqslant \frac{L_{0_{\max }}}{R_{0}}
$$

Since from Table 4, $\gamma_{p}=\beta_{e}-\alpha$, the constraints ( $\left.29 \mathrm{~b}, \mathrm{c}\right)$ can be rewritten as

$$
\gamma_{p \min }=\beta_{e}-\alpha_{\max } \leqslant \gamma_{p} \leqslant \gamma_{0}-\varepsilon=\gamma_{p \max },
$$

which indicate the equivalent design freedom in $\gamma_{p}$.

Note that if $\gamma_{0}$ is chosen constant, then $\alpha_{\min }, \alpha_{\max }$, and $\gamma_{p_{\max }}$ are constants. If furthermore $\beta_{e}$ is constant, then so is $\gamma_{p_{\min }}$.

Let us now consider one way of choosing $2_{0}$, namely by relating it to the borrower's expected real net income rate

$$
g_{e}=I_{0} E_{\sigma_{e}},
$$

say, by

$$
\mathcal{Q}_{0}=p \mathcal{g}_{e},
$$

where

$$
p=p_{0} E_{s}, \text { say, }
$$

is the largest fraction of income that the borrower allocates for mortgage payments. It then follows that

$$
R_{0}=p_{0} I_{0}, \quad \gamma_{0}=s+\sigma_{e},
$$

and

$$
\frac{L_{0}}{I_{0}}=p_{0} G_{\alpha_{\max }}(0)
$$


For example, $p_{0}$ could be chosen in the range 15-35\%. $s$ controls the fraction of payment/income, and could be chosen conservatively small negative (say $-1 \%$ per year), through to small positive. $\sigma_{e}$ could also be estimated conservatively, since its value may be difficult to estimate. More simply, $\gamma_{0}$ could be chosen small negative (say $-1 \%$ per year), or 0 , or small positive (say 1 or $2 \%$ per year) according as $q_{e}$ is expected to have a small decrease, to remain constant or is confidently expected to increase.

It is clear from (29) that the tightness of the design depends on the ratio $L_{0} / L_{0_{\max }}$ If $L_{0}=L_{0_{\text {max }}}$, then

$$
\alpha=\alpha_{\min }=\alpha_{\max }, R=R_{0}, \quad \gamma_{p}=\gamma_{0}-\varepsilon, \quad \gamma_{0}-2 \varepsilon \leqslant \gamma \leqslant \gamma_{0},
$$

and no freedom is then possible in the choice of $\alpha$ (or $\gamma_{p}$ ). On the other hand, if $L_{0} / L_{0_{\max }}<1$, then there remains some freedom of choice in $\alpha$ satisfying $(29 \mathrm{~b}, \mathrm{c})$ (or equivalently in $\gamma_{p}$ satisfying $(30 \mathrm{a}, \mathrm{b})$ ). The smaller $L_{0} / L_{0_{\max }}$, the greater is this degree of freedom. This is illustrated in Tables 6(a), (b), (c) for cases when $\gamma_{0}$ is chosen constant. (The values given can be checked using Table 3 ).

TABLE 6. Dependence of design parameters on $L_{0} / L_{0_{\max }}$ for $T=25$ years, $p_{0}=\frac{1}{4}, \beta_{e}=3 \%$ per year, $\gamma_{0}$ constant.

(a) Example 1. $\gamma_{0}-\varepsilon=\gamma_{p_{\max }}=-3 \%$ per year (e.g. $\left(\gamma_{0}, \varepsilon\right)=(-2,1),(-1,2)$, or $(0,3) \%$ per year) so that $\alpha_{\min }=6 \%$ per year, $G_{\alpha_{\min }}(0)=12.95$ years.

\begin{tabular}{|l|c|c|c|c|}
\hline$L_{0} / L_{0_{\max }}$ & 1 & .835 & .709 & .612 \\
$G_{\alpha_{\max }(0) \text { (years) }}$ & 12.95 & 10.81 & 9.18 & 7.92 \\
$\alpha_{\max }(\% /$ year $)$ & 6 & 8 & 10 & 12 \\
$\gamma_{p_{\min }}(\% /$ year $)$ & -3 & -5 & -7 & -9 \\
$L_{0} / I_{0}$ (years) & 3.24 & 2.70 & 2.295 & 1.98 \\
\hline
\end{tabular}

(b) Example 2. $\gamma_{0}-\varepsilon=\gamma_{p_{\max }}=-1 \%$ per year (e.g. $\left(\gamma_{0}, \varepsilon\right)=(0,1),(1,2)$, or $(2,3) \%$ per year) so that $\alpha_{\min }=4 \%$ per year, $G_{\alpha_{\min }}(0)=15.80$ years.

\begin{tabular}{|l|c|c|c|c|}
\hline$L_{0} / L_{0 \max }$ & 1 & .822 & .684 & .581 \\
$G_{\alpha_{\max }}(0)$ (years) & 15.80 & 12.95 & 10.81 & 9.18 \\
$\alpha_{\max }$ (\%/year) & 4 & 6 & 8 & 10 \\
$\gamma_{p_{\min }}$ (\%/year) & -1 & -3 & -5 & -7 \\
$L_{0} / I_{0}$ (years) & 3.95 & 3.24 & 2.70 & 2.295 \\
\hline
\end{tabular}

(c) Example 3. $\gamma_{0}-\varepsilon=\gamma_{p_{\max }}=1 \%$ per year (e.g. $\left(\gamma_{0}, \varepsilon\right)=(2,1),(3,2)$, or $(4,3) \%$ per year) so that $\alpha_{\min }=2 \%$ per year, $G_{\alpha_{\min }}(0)=19.68$ years.

\begin{tabular}{|l|c|c|c|c|}
\hline$L_{0} / L_{0_{\max }}$ & 1 & .803 & .658 & .549 \\
$G_{\alpha_{\max }(0) \text { (years) }}$ & 19.68 & 15.80 & 12.95 & 10.81 \\
$\alpha_{\max }(\% /$ year) & 2 & 4 & 6 & 8 \\
$\gamma_{p_{\text {min }}}$ (\%/year) & 1 & -1 & -3 & -5 \\
$L_{0} / I_{0}$ (years) & 4.92 & 3.95 & 3.24 & 2.70 \\
\hline
\end{tabular}


Regarding the final choice of $\alpha$ to satisfy $\left(29 \mathrm{~b}, \mathrm{c}\right.$ ) (in the case $L_{0} / L_{0_{\max }}<1$ ) there are a number of possible approaches.

(1) Choose $\alpha=\alpha_{\max }$ (most conservatively).

(2) Choose $\alpha=\alpha_{\min }$ (most radically).

(3) Choose $\alpha=\frac{1}{2}\left(\alpha_{\min }+\alpha_{\max }\right)$.

(4) Choose $\alpha$ as an optimal solution-i.e. the solution to some 'appropriate well-posed' optimization problem. It should be noted here that Cohn and Fischer [4, pages 48-52] considered 'criteria for evaluating mortgage instrument design'. However, these criteria are only semi-quantitative, and are by no means universally accepted (see Thygerson [10]). The optimization of $\alpha$ therefore remains an open question.

In many cases (for example if $\alpha_{\min }, \alpha_{\max }$ are constants), it will be possible (and may be desirable for simplicity) to restrict the analysis to the class of SCM's, and to choose a constant value of the 'parameter' $\alpha$.

More simply, if the examples in Table 6 are typical of long-term mortgages, then choosing $\alpha$ to be a constant within the range from 4\% per year (radical) to $8 \%$ per year (conservative) would be an appropriate rough and ready rule. However, we should be reminded that these values are very sensitive to $\beta_{e}$ and $\varepsilon$.

\section{Conclusions}

The following conclusions are based on the preceding analysis of the continuous mortgage (CM). In so far as any mortgage is either a CM, or can be regarded as a sequence of $\mathrm{CMs}$, they can be applied to any mortgage.

(Cl) In order to make satisfactory mortgage design possible it is necessary that the real interest rate $\beta$ should satisfy a restraint of the form

$$
\left|\beta-\beta_{e}\right| \leqslant \varepsilon \quad\left(\beta_{e}, \varepsilon \text { constants }\right),
$$

for a suitably small $\varepsilon$ (Section 7).

(C2) The CM offers a continuum of satisfactory mortgage instruments provided the restraints (25a) on $\beta$ and (29) on the target design are imposed (Sections $7,8)$.

(C3) The actual real payment rate 2 of the $\mathrm{CM}$ will deviate from the planned (target) $2_{p}$ by the 'deviation factor' $E_{\beta-\beta_{\varepsilon}}$ which is exponentially dependent on the deviation $\beta-\beta_{e}$ (Section 7).

(C4) The choice $\varepsilon \sim 1 \%$ per year is a practical compromise between allowing $\beta$ to change unrestrictedly to accommodate the supply demand for money in real terms, and minimizing the design deviation $\beta-\beta_{e}$ (cf. Table 5, Section 7). 
(C5) The restraints (29) on the initial loan $L_{0}$, and the control function $\alpha$ are chosen to ensure that 2 has an appropriate upper bound $2_{0}(27)$ which can either be chosen conservatively or related to the borrower's expected real income ( $3 \mathrm{lb}$ ).

(C6) The mortgage should be indexed, i.e. $\beta$ should be assigned rather than the nominal interest rate $l$.

This is necessary to ensure (25a) whenever the inflation rate is volatile.

(C7) Changes in the assignment of $\beta$ (satisfying (25a)) should only be made deliberately to reflect changes in real money pressures. Such changes should be made by an independent (e.g. government) agency.

These changes in $\beta$ are expected to be small and infrequent. This is in contrast with the current common practice of assigning $l(=\beta+\lambda)$ and not letting it accommodate sufficiently or sufficiently rapidly to changes in the inflation rate $\lambda$, so that $\beta$ is fluctuating almost in opposition to the whims of inflation. This can even result in $\beta$ becoming negative [7, page 91].

(C8) Assigning $\beta$ rather than $l$ will also focus attention on the real mortgage functions, and therefore encourage the acceptance of CM design with $\alpha \sim \beta$, rather than the unsatisfactory $\alpha \sim l$ (as with the standard mortgage (SM) and standard variable rate mortgage (SVRM)).

(C9) The possible optimization of the target design, determined by $\alpha$, remains an open question (Section 8).

(C10) The SCM (with $\alpha$ constant) may be sufficiently general for most practical purposes. A choice of constant $\alpha$ in the range $4-8 \%$ per year would be suitable for a 'typical' long term mortgage (Section 8).

\section{Appendix 1 - Method of repayments}

Our continuous model requires that the repayments are made continuously at the rate $Q(t)$. However, as a practical scheme, the repayments could be made in lumps at convenient regular intervals of duration $\Delta t$ (e.g. one month). Allowing for the continuous charging of interest, the repayment rate $Q(t)$ is then equivalent to making lumped payments $P\left(\tau_{k}\right)$ at times $\tau_{k}=k \Delta t, k=1,2, \ldots, \bar{T}(=T / \Delta t$, assumed an integer) as indicated in both nominal and real terms in Table 7 , where

$$
\tau_{0}=0, \quad \int_{0}^{\Delta t} e^{\alpha u} d u= \begin{cases}\frac{1}{\alpha}\left(e^{\alpha \Delta t}-1\right) & \text { if } \alpha \neq 0 \\ \Delta t & \text { if } \alpha=0\end{cases}
$$


TABLE 7. Equivalent lumped payments.

\begin{tabular}{|c|c|c|}
\hline $\begin{array}{c}\text { Mortgage } \\
\text { type }\end{array}$ & $\begin{array}{c}\text { Nominal terms } \\
P\left(\tau_{k}\right)\end{array}$ & $\begin{array}{c}\text { Real terms } \\
\mathcal{P}\left(\tau_{k}\right)=P\left(\tau_{k}\right) E_{-\lambda}\left(\tau_{k}\right)\end{array}$ \\
\hline $\mathrm{GM}$ & $\int_{\tau_{k-1}}^{\tau_{k}} Q(t) \frac{E_{l}\left(\tau_{k}\right)}{E_{l}(t)} d t$ & $\int_{\tau_{k-1}}^{\tau_{k}} \mathcal{2}(t) \frac{E_{\beta}\left(\tau_{k}\right)}{E_{\beta}(t)} d t$ \\
\hline $\mathrm{CM}$ & $Q\left(\tau_{k}\right) \int_{\tau_{k-1}}^{\tau_{k}} \frac{E_{\alpha}\left(\tau_{k}\right)}{E_{\alpha}(t)} d t$ & $2\left(\tau_{k}\right) \int_{\tau_{k-1}}^{\tau_{k}} \frac{E_{\alpha}\left(\tau_{k}\right)}{E_{\alpha}(t)} d t$ \\
\hline $\mathrm{SCM}$ & $Q\left(\tau_{k}\right) \int_{0}^{\Delta t} e^{\alpha u} d u$ & $2\left(\tau_{k}\right) \int_{0}^{\Delta t} e^{\alpha u} d u$ \\
\hline
\end{tabular}

If $\alpha$ is prescribed, and $\beta$ is assigned (Section $9(\mathrm{C} 6)$ ), then $\mathscr{P}\left(\tau_{k}\right)$ can be calculated from Table 7. However, there will be a delay in being able to calculate the actual payment $P\left(\tau_{k}\right)=E_{\lambda}\left(\tau_{k}\right) \mathscr{P}\left(\tau_{k}\right)$ due to the delay in establishing the price level (and hence $\lambda$ ) right up to the current time $\tau_{k}$. This can be adequately dealt with either

(a) by accepting $\lambda$ values slightly in arrears (1-3 months, say) throughout the mortgage, or

(b) by estimating the current $\lambda$ value, and making any necessary correction to the next payment (or group of payments).

\section{Appendix 2 - Comparison of effective annual interest rates}

Here we illustrate the dependence of the effective annual interest rate on the compounding frequency $m$ (times/year) and the nominal annual interest rate $S$. We note that $S=S_{1}$, which corresponds to compounding annually, $S_{m}$ corresponds to compounding $m$ times/year, whereas $S_{\infty}=e^{S}-1$ corresponds to compounding continuously.

TABLE 8. Values of the $m$-yearly effective annual interest rate $S_{m}=(1+S / m)^{m}-1$ (in $\%$ ).

\begin{tabular}{|l|c|c|c|c|c|c|c|}
\hline $\begin{array}{l}S \\
\text { (in \%) }\end{array}$ & 1 & 2 & 4 & 12 & 52 & 365 & $\infty$ \\
\hline 1 & 1 & 1.003 & 1.004 & 1.005 & 1.005 & 1.005 & 1.005 \\
2 & 2 & 2.010 & 2.015 & 2.018 & 2.020 & 2.020 & 2.020 \\
5 & 5 & 5.063 & 5.095 & 5.116 & 5.124 & 5.127 & 5.127 \\
10 & 10 & 10.25 & 10.38 & 10.47 & 10.51 & 10.52 & 10.52 \\
15 & 15 & 15.56 & 15.87 & 16.08 & 16.16 & 16.18 & 16.18 \\
20 & 20 & 21.00 & 21.55 & 21.94 & 22.09 & 22.13 & 22.14 \\
30 & 30 & 32.25 & 33.55 & 34.49 & 34.87 & 34.97 & 34.99 \\
\hline
\end{tabular}


The extent to which $S_{m}$ approximates $S_{\infty}$ can be measured by

$$
\frac{S_{\infty}-S_{m}}{S_{\infty}-S_{1}}=\frac{1}{m}\left[1+\frac{2}{3}\left(1-\frac{1}{m}\right) S+O\left(S^{2}\right)\right] \text {. }
$$

For example, for $m=12$ this ratio approximates $\frac{1}{12}\left(1+\frac{11}{18} S\right)$, so that monthly compounding is substantially continuous as compared with annual compounding. This is also clear from Table 8.

\section{References}

[1] R. Anderson and D. R. Lessard, "Price-level-adjusted mortgages in Brazil", in [8] (1975), 115-129.

[2] J. Black, "A new system for mortgages", Lloyds Bank Review III (1974), 9-16.

[3] R. A. Cohn and D. R. Lessard, "Recent research on indexation and the housing market", $J$. Finance 31 (1976), 403-413.

[4] R. A. Cohn and S. Fischer, "Alternative mortgage designs", in [8] (1975), 47-74.

[5] W. F. Lee, "Some alternatives to conventional farm mortgage loan repayment plans", Canadian Farm Economics (1979).

[6] D. R. Lessard and F. Modigliani, "Inflation and the housing market: problems and solutions", in [8] (1975), 13-45.

[7] F. Modigliani, "Some economic implications of the indexing of financial assets with special reference to mortgages", in The new inflation and monetary policy (ed. M. Monti), (Macmillan, London, 1976), 90-116.

[8] F. Modigliani and D. R. Lessard (eds.), New mortgage designs for stable housing in an inflationary environment (Federal Reserve Bank of Boston Conference Series No. 14, 1975).

[9] Tan, Soo Tang, "An iterative procedure for computing the effective rates of interest", Bull. Inst. Math. Appl. 15 (1979), 70-71.

[10] K. J. Thygerson, “Discussion”, in [8] (1975), 81-86.

[11] D. Tucker, “The variable-rate graduated-payment mortgage", Real Estate Review (1975).

[12] R. W. R. White, "The concept of a constant value unit of account", Reserve Bank of New Zealand Bulletin (Nov., Dec. 1979). 